\title{
Molecular importance of prawn large heat shock proteins 60, 70 and 90
}

\begin{abstract}
Considering the importance of heat shock proteins (HSPs) in the innate immune system of prawn, a comparative molecular approach was proposed to study the crustacean large HSPs 60, 70 and 90. Three different large HSPs were identified from freshwater prawn Macrobrachium rosenbergii (Mr) cDNA library during screening. The structural and functional characteristic features of HSPs were studied using various bioinformatics tools. Also, their gene expression and mRNA regulation upon various pathogenic infections was studied by relative quantification using 2-qeT method. MrHSP60 contains a long chaperonin 60 domain at $46 i \ddot{~} 547$ which carries a chaperonin 60 signature motif between 427 and 438, whereas MrHSP70 contains a long HSP70 domain at 21ї 624 and MrHSP90 carries a HSP90 domain at 1881719 . The two dimensional analysis showed that MrHSP60 contains more amino acids (52\%) in helices, whereas MrHSP70 (40.6\%) and MrHSP90 (51.8\%) carried more residues in coils. Gene expression results showed significant $(\mathrm{P}<0.05)$ expression of MrHSP60, 70 and 90 in haemocyte, gill and hepatopancreas, respectively. Further, the expression level was up-regulated upon bacterial (Aeromonas hydrophilla and Vibrio harveyi) and viral [white spot syndrome virus (WSSV) and M. rosenbergii nodo virus $(\mathrm{MrNV})]$ infections during various time periods. The gene expression results exhibited the potential involvement of these three HSPs in the immune system of prawn. The study indicated the potentiality of these molecules, thereby protecting cells against pathogens as well as severe cellular and environmental stresses in crustaceans.
\end{abstract}

Keyword: Heat shock proteins; Macrobrachium rosenbergii; Bioinformatics; Gene expression; Pathogen 